\title{
APENDICECTOMIA VIDEOLAPAROSCÓPICA: ANÁLISE PROSPECTIVA DE 300 CASOS
}

\section{Laparoscopic appendectomy: prospective study of 300 cases}

\author{
André Luiz Gonçalves de OLIVEIRA, André Takashi OTI, Edson Yuzur YASOJIMA, \\ Helder Costa IKEGAMI, Pedro Antônio Mufarrej HAGE, Tárik Olívar de Nunes VALENTE
}

ABCDDV/595

Oliveira ALG, Oti AT, Yasojima EY, Ikegami HC, Hage PAM, Valente TON. Apendicectomia videolaparoscópica: análise prospectiva de 300 casos. ABCD Arq Bras Cir Dig. 2008;21(2):69-72

RESUMO - Racional - A apendicectomia é o tratamento de escolha para os casos de apendicite aguda e a evolução das técnicas operatórias faz da videocirurgia boa opção terapêutica. Objetivo - Analisar os achados clínicos e demográficos de 300 pacientes com apendicite aguda, bem como a viabilidade e evolução intra e pós-operatória das apendicectomias videolaparoscópicas neles realizadas. Métodos - Foram estudados todos os pacientes com diagnóstico confirmado de apendicite submetidos à cirurgia videolaparoscópica no Hospital da Beneficência Nipo-Brasileira da Amazônia, no período de agosto de 2000 a julho de 2008, com a utilização de protocolo para a coleta de dados. Ele incluía dados demográficos, aspectos clínicos e de achados físicos para o diagnóstico, resultados do leucograma, aspectos técnicos do procedimento cirúrgico, posição do apêndice, fase de inflamação apendicular, operações associadas realizadas, tempo operatório, complicações pós-operatórias e a recuperação pós-operatória. Resultados - Observou-se que a maioria dos pacientes encontrava-se entre a segunda e terceira décadas e a apendicite era mais comum nos homens. Dor em fossa ilíaca direita, febre, náuseas e vômitos ocorreu em $65,44 \%$ e $84,29 \%$ apresentaram leucocitose. A disposição anatômica apendicular mais encontrada foi pélvica em $84,29 \%$ dos casos, seguida da retrocecal com 7,14\%, da retrocecal-subserosa com 5,71\% e da subserosa com $2,86 \%$. Em $10 \%$ dos casos foram realizadas operações associadas. A média de tempo operatório foi de 55 minutos, com taxa de conversão de $2 \%$. Não foram observadas complicações intra-operatórias e as pós-operatórias foram de 7,1\%. O tempo de internação ficou na média de 48,5 horas. O retorno às atividades habituais ocorreu em média de 5 dias. Conclusão - A apendicectomia videocirurgia é procedimento seguro, eficaz com restabelecimento rápido dos pacientes, devendo ser sempre pensada quando for tratado quadro de apendicite aguda em qualquer idade.

DESCRITORES - Apendicite. Apendicectomia. Videolaparoscopia.

\section{INTRODUÇÃO}

Desde 1886, quando foi descrito pela primeira vez por Fitz, a apendicectomia é o procedimento de escolha para os casos de apendicite aguda².

Ao longo do tempo, as técnicas operatórias foram evoluindo, e a videocirurgia surgiu para minimizar os efeitos dos procedimentos operatórios ${ }^{2,5,7,10}$.

Em 1982, Kurt Semm, em Kiel, Alemanha, realizou a primeira apendicectomia por via laparoscópica, embasado no sucesso da utilização da videocirurgia para outros órgãos como a vesícula biliar, com bom campo operatório ${ }^{5,710}$. Este método proporciona alguns benefícios relevantes para os pacientes, tais como, menor tempo de internação, melhor pós-operatório, menor índice de infecção da ferida operatória, retorno precoce as atividades habituais, além de proporcionar completa visualização da cavidade abdominal ${ }^{1,2,4,5,8,9}$.

Apesar desses benefícios, muitos ainda não a consideram como procedimento padrão devido à falta de estudos para seguimento de seus resultados à longo prazo e pelo elevado

Trabalho realizado no Serviço de Cirurgia Geral do Hospital da Beneficência Nipo-Brasileira da Amazônia, Belém, PA, Brasil.

Endereço para correspondência: Tárik Olívar de Nunes Valente - e-mail: tarikolivar@globo.com custo financeiro e recursos tecnológicos necessários ${ }^{2,3,6}$.

O presente estudo tem por objetivo relatar os resultados obtidos em 300 casos de apendicite aguda avaliados prospectivamente, tratados com apendicectomia videolaparoscópica e seguidos a longo prazo.

\section{MÉTODOS}

Foram estudados 300 casos de apendicectomias videolaparoscópicas que procuraram atendimento no Hospital da Beneficência Nipo-Brasileira da Amazônia, no período de agosto de 2000 à julho de 2008.

Após o diagnóstico confirmado de apendicite por exame físico e leucograma, era iniciado o preenchimento de protocolo próprio e os preparativos pré-operatórios. O protocolo incluía dados demográficos, aspectos clínicos e de achados físicos para o diagnóstico, resultados do leucograma, aspectos técnicos do procedimento cirúrgico, posição do apêndice, fase de inflamação apendicular, operações associadas realizadas, tempo operatório, complicações pós-operatórias e a recuperação pós-operatória.

A técnica cirúrgica utilizou 3 portais de acesso à cavidade abdominal, localizados em: região mediana supra umbilical (trocarte de $10 \mathrm{~mm}$ ), transição do flanco esquerdo para a fossa ilíaca esquerda (trocarte de $10 \mathrm{~mm}$ ) e em fossa ilíaca esquerda (trocater de $5 \mathrm{~mm}$ ) (Figura 1). 


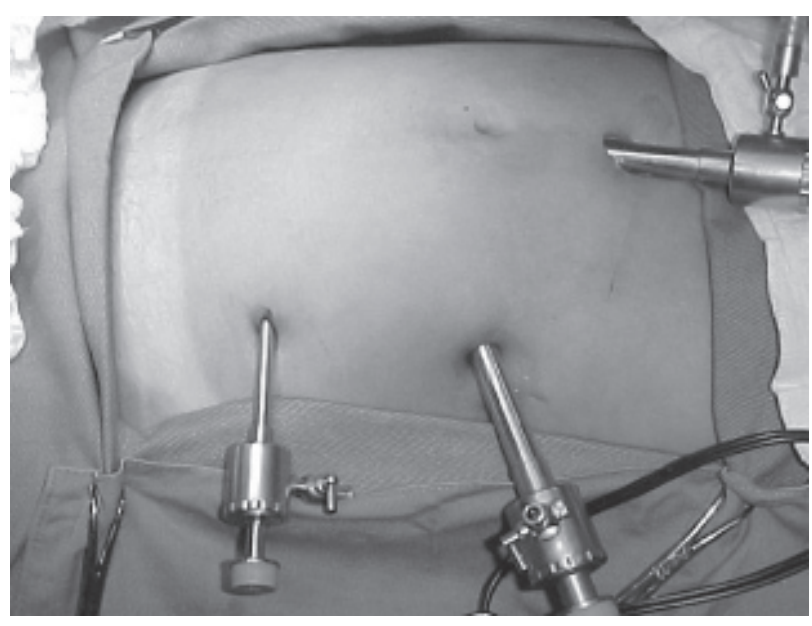

FIGURA 1 - Posição dos trocartes

O aparelho de videolaparoscopia utilizado foi da marca Olympus $^{\circledR}$, contendo monitor de vídeo modelo OEV 202; micro-câmera modelo OTU-S4; insuflador de $\mathrm{CO}^{2}$ automático, acoplado a cilindro de 500 libras de $\mathrm{CO}^{2}$; fonte de luz de xenônio, modelo CLV-S20 e gravador de mídia DVD.

No período em que permaneciam internados, os pacientes eram acompanhados pelos membros da equipe cirúrgica, que faziam as evoluções e as registravam no protocolo.

Com os dados obtidos, foi criado banco de dados Microsoft Access $2000^{\circledR}$, onde foram armazenadas as informações referentes a cada paciente.

Os resultados obtidos foram submetidos à análise estatística, aplicando-se o teste paramétrico do Qui-quadrado e o não paramétrico de Kruskal-Wallis com $\mathrm{P}<0,05$.

\section{RESULTADOS}

A distribuição dos pacientes segundo o sexo e a faixa etária está disposta na Tabela 1. Observa-se que a maioria encontrava-se entre a segunda e terceira décadas e mais comum nos homens.

TABELA 1 - Distribuição dos pacientes segundo o sexo e a faixa etária

\begin{tabular}{lcccccc}
\hline \multirow{2}{*}{ FAIXA ETÁRIA } & \multicolumn{2}{c}{ MASCULINO } & \multicolumn{2}{c}{ FEMININO } & \multicolumn{2}{c}{ TOTAL } \\
& $\mathbf{N}^{\mathbf{0}}$ & $\mathbf{\%}$ & $\mathbf{N}^{\mathbf{0}}$ & $\mathbf{\%}$ & $\mathbf{N}^{\mathbf{0}}$ & $\mathbf{\%}$ \\
\hline $0-09$ & 5 & 2,8 & 4 & 3,3 & 9 & 3 \\
$10-19$ & 93 & 51,6 & 42 & 35 & 135 & 45 \\
$20-29$ & 37 & 20,5 & 26 & 21,6 & 63 & 21 \\
$30-39$ & 22 & 12,2 & 16 & 13,3 & 38 & 12,7 \\
$40-49$ & 10 & 5,6 & 27 & 22,5 & 37 & 12,3 \\
$50-59$ & 10 & 5,6 & 3 & 2,5 & 13 & 4,3 \\
60 e + & 3 & 1,7 & 2 & 1,8 & 5 & 1,7 \\
\hline TOTAL & $\mathbf{1 8 0}$ & $\mathbf{1 0 0}$ & $\mathbf{1 2 0}$ & $\mathbf{1 0 0}$ & $\mathbf{3 0 0}$ & $\mathbf{1 0 0}$ \\
\hline
\end{tabular}

FONTE: Hospital da Beneficência Nipo-Brasileira da Amazônia

O quadro clínico de dor em fossa ilíaca direita, febre, náuseas e vômitos ocorreu em $65,44 \%$ dos pacientes e
$84,29 \%$ deles apresentaram leucocitose.

Em relação ao exame físico 97,14\% apresentaram dor à palpação, seguido de $85,71 \%$ com sinal de Blumberg positivo e $11,43 \%$ apresentaram plastrão.

A disposição anatômica apendicular mais encontrada foi pélvica em $84,29 \%$ dos casos, seguida da retrocecal com $7,14 \%$, da retrocecal-subserosa com $5,71 \%$ e da subserosa com $2,86 \%$.

Em relação à fase de inflamação apendicular $100 \%$ dos casos foram de apendicite aguda, nas mais diferentes fases de evolução (Tabela 2).

TABELA 2 - Distribuição dos pacientes segundo o sexo e a fase da apendicite

\begin{tabular}{lcccccc}
\hline \multirow{2}{*}{$\begin{array}{l}\text { A PEND ICITE } \\
\text { AGUDA }\end{array}$} & \multicolumn{2}{c}{ MASCULINO } & \multicolumn{2}{c}{ FEMININO } & \multicolumn{2}{c}{ TOTAL } \\
\hline Catarral & $\mathbf{N}^{\mathbf{0}}$ & $\mathbf{\%}$ & $\mathbf{N}^{\mathbf{0}}$ & $\mathbf{\%}$ & $\mathbf{N}^{\mathbf{0}}$ & $\mathbf{\%}$ \\
Supurada & 117 & 65 & 87 & 72,5 & 204 & 68 \\
Gangrenada & 35 & 19,4 & 13 & 10,8 & 48 & 16 \\
Perfurativa & 12 & 6,7 & 12 & 10 & 24 & 8 \\
\hline T O T A L & 16 & 8,9 & 8 & 6,7 & 24 & 8 \\
\hline
\end{tabular}

FONTE: Hospital da Beneficência Nipo-Brasileira da Amazônia

Em 10\% dos casos foram realizadas operações associadas à apendicectomia: colecistectomia, ooforectomia e salpingectomias.

A média de tempo operatório foi de 55 minutos, com taxa de conversão de $2 \%$. Não foram observadas complicações intra-operatórias. No entretanto, a taxa de complicações pós-operatórias foi de $7,1 \%$ e o tempo de internação ficou na média de 48,5 horas. $\mathrm{O}$ retorno às atividades habituais ocorreu em média de 5 dias.

\section{DISCUSSÃO}

Com a finalidade de obter-se melhores resultados através de procedimentos menos invasivo, está crescendo cada vez mais o aprimoramento das técnicas videolaparoscópicas, utilizando-se deste método para resolução de doenças cirúrgicas em todos os órgãos da cavidade abdominal.

A respeito da epidemiologia da apendicite e apendicectomia nos Estados Unidos, a literatura refere não haver diferença significativa na incidência de apendicite entre os sexos masculino e feminino, entretanto, o risco de ser submetido a apendicectomia no decurso da vida é $12 \%$ maior em mulheres ${ }^{2}$. No presente estudo foi reconhecida predominância do sexo masculino, com a faixa etária de adolescentes e adultos jovens prevalecendo em relação às demais.

Estudos demonstraram que a apendicectomia videolaparoscópica em crianças e adolescentes - para as formas complicadas de apendicite -, não apresentou diminuição significativa do tempo de permanência hospitalar ou benefícios intra-operatórios, e teve maior incidência de formação de abscessos intra-abdominais como outras complicações pós-operatórias ${ }^{4}$. Este fato, contrapõe-se ao 
presente estudo, pois, o procedimento de apendicectomia videolaparoscópica, quando realizado em crianças e adolescentes, não apresentou aumento de complicações.

$\mathrm{Na}$ maioria significativa desta a dor localizada em fossa ilíaca direita foi a mais comum, seguida do quadro clássico de apendicite aguda com dor epigástrica irradiando para fossa ilíaca direita, acompanhada de febre. Náuseas e vômitos foram sintomas freqüentes ao quadro clínico apresentado, já sintomas diarréicos foram referidos em poucos casos.

Para se estabelecer o diagnóstico de apendicite no estudo em vigência foi relevante a história clínica e exame físico, utilizando-se de três variáveis principais: a presença de dor, a irritação peritoneal e a presença de massa ou plastrão indicando bloqueio por alças intestinais ou omento no apêndice inflamado.

A leucometria foi utilizada como recurso laboratorial para auxiliar o diagnóstico e na grande maioria da casuística obteve-se leucocitose com desvio à esquerda.

A localização apendicular mais freqüente foi a retrocecal intraperitoneal, ocorrendo em $2 / 3$ dos pacientes, seguido da pélvica. Esta posição pode ser diferenciada clinicamente ela dor suprapúbica e disúria. Em apenas 5\% dos casos a ponta do apêndice era retroperitoneal e ocasionava dor lombar ou no flanco com alterações urinárias freqüentes e dor testicular. $\mathrm{O}$ apêndice cecal pode ser longo, variando de 3 a $20 \mathrm{~cm}$ de comprimento, e sua ponta pode localizarse em quadrante inferior esquerdo, ocasionando dor na referida topografia. A localização da dor é anômala na presença de má rotação intestinal e situs inversus ${ }^{5}$. No presente estudo a localização da dor apendicular mais freqüente foi a pélvica.

Houve casos de apêndices retrocecais, retrocecalsubserosos e subserosos, fatos estes que não inviabilizaram a realização da apendicectomia videolaparoscópica, mas motivaram a elevação do tempo operatório por requerer dissecção mais cuidadosa e trabalhosa.

Este estudo apresenta a realização da apendicectomia videolaparoscópica na apendicite aguda em suas diferentes fases (catarral, supurada, gangrenada e perfurada). Entretanto, ocorreram 3 casos não agudos onde a videolaparoscopia diagnóstica indicou as apendicectomias. Nestes casos o apêndice apresentava características macroscópicas normais e através do resultado de exame histopatológico foi detectado apendicite crônica em 2 casos e apêndice normal em 1 caso.

Entre os fatores que contribuíram para média de tempo operatório elevada, quando comparada com a literatura pesquisada, foram a posição apendicular mais difícil de acesso, fases mais avançadas da apendicite, a realização de operações associadas, a utilização de nó de endoloop de confecção manual e problemas técnicos no equipamento. A elevação do tempo operatório é bastante influenciada pela curva de aprendizado da equipe cirúrgica, uma vez que o procedimento videolaparoscópico requer treinamento mais específico do cirurgião do que os procedimentos convencionais. Este fato foi responsável, na literatura pesquisada, pela variação no que diz respeito ao tempo operatório, com tempo entre 20 a 104 minutos. Foi observado que quanto maior a casuística menor era o tempo operatório, confirmando a influência da curva de aprendizado dos profissionais que realizam o procedimento ${ }^{6,9}$.

Outro fator de relevância foi a realização de operações associadas, que foram realizadas no presente estudo em topografia próxima ou distante do sítio de infecção primário, utilizando os mesmos portais e outros adicionais.

O tempo de permanência hospitalar dos pacientes no presente estudo, sofreu influência direta das formas complicadas de apendicite associada com outros fatores inerentes ao procedimento cirúrgico, como a presença de infecção pós-operatória. Apesar desta influência, o tempo de permanência hospitalar foi pequeno, associado a rápido retorno as suas atividades habituais. Alguns autores não consideram significativa a redução do tempo de internação, nem o retorno precoce as atividades normais ${ }^{1,3}$. Outros consideraram que ele é reduzido e ocorre devido a melhor pós-operatório, com menos dor, menor utilização de analgesia parenteral, precoce ingestão de alimentos sólidos e retorno precoce as atividades habituais ${ }^{8,10}$.

Os achados de infecção de ferida cirúrgica no pósoperatório são reduzidos nos pacientes submetidos a este procedimento. Contudo, há descrição da presença de abscesso intra-abdominal na literatura ${ }^{6}$. No presente estudo foi observado índice pequeno de infecções. No entretanto, foram observadas complicações intra-operatórias que inviabilizaram o procedimento videolaparoscópio, como peritonite e processos aderenciais intensos em 6 casos.

Além dos casos de infecção de ferida cirúrgica, foi observado 1 caso de abdome agudo obstrutivo no $7^{\circ}$. dia de pós-operatório, tendo sido submetido à laparotomia exploradora para resolução dos processos aderenciais com ressecção de alça de intestino delgado.

Para que não houvesse contato do órgão inflamado com a ferida operatória e assim reduzir as taxas de infecção de ferida, foi utilizado o endobag, material plástico em fundo de saco, no qual era inserido o apêndice cecal para ser retirado da cavidade abdominal. Os casos que apresentaram infecção de ferida operatória houve o rompimento do endobag, ocorrendo o contato do órgão infectado com a ferida, ficando a infecção restrita à incisão mediana supraumbilical, por onde era retirado o órgão.

\section{CONCLUSÃO}

A apendicectomia videocirurgia é procedimento seguro, eficaz com restabelecimento rápido dos pacientes, devendo ser sempre pensada quando for tratado quadro de apendicite aguda em qualquer idade. 
Yasojima EY, Valente TON. Laparoscopic appendectomy: prospective study of 300 cases. ABCD Arq Bras Cir Dig. 2008;21(2):69-72

ABSTRACT - Background - Appendectomy is the chosen treatment for patients with acute appendicitis and laparoscopic approach for these cases is an option to have in mind. Aim - To analyze the clinical aspects, technical results and the evolution during and after the operation in 300 cases of laparoscopic appendectomy. Methods - All the patients with diagnosed appendicitis were submitted to laparoscopic appendectomy on Beneficencia Nipo-Brasileira Hospital of Amazon, between 2000 (august) and 2008 (july), using a specific protocol. Results - There was a predominance in teen and young adults, male sex, having a classic clinical finding of acute appendicitis in $65,44 \%$ of the cases, leucocytosis in $84,29 \%$ and by anatomic pelvic site of vermiform appendix in 84,29 . The mean time taken by the operation was 55 minutes with a conversion rate of $7,1 \%$. In hospital period was 48,5 hours with 5 days to return to normal activities. Conclusion - The laparoscopic appendectomy can be useful in male and female, at any age, in all the phases of acute appendicitis and in all anatomic sites of vermiform appendix.

HEADINGS - Appendicitis. Appendectomy. Videosurgery

\section{REFERÊNCIAS}

1. Frazee RC, Bohannon WT. Laparoscopic appendectomy for complicated appendicitis. Arch. Surg. 1996; 131(5):509-11.

2. Fritts LL, Orlando R. Laparoscopic appendectomy: a safety and cost analysis. Arch. Surg. 1993; 128(5):521-5.

3. González Moreno S, Shmookler BM, Sugarbaker PH. Appendiceal mucocele: contraindication to laparoscopic appendectomy. Surg. Endosc. 1998; 12(9):1177-9.

4. Horwitz JR, Custer MD, May BH, Mehall JR, Lally KP. Should laparoscopic appendectomy be avoided for complicated appendicitis in children? J. Pediat. Surg. 1997; 32(11):1601-03.

5. Klinger A, Henle KP, Beller S, Rechner J, Zerz A,Wetscher GJ et al. Laparoscopic appendectomy does not change the incidence of postoperative infectious complications. Am. J. Surg. 1998; 175(3):232-5.
6. MerhoffAM, Merhoff GC, Franklin ME. Laparoscopic versus open appendectomy. Am. J. Surg. 2000; 179(5):375-8.

7. del Rio Martin JV, Ashraf Memon M. Justificación de la apendicectomía laparoscópica. Rev. Esp. Enferm. Dig. 1999; 91(6):447-55.

8. Rüedi TP. Apendicite aguda. In: Coelho JCU. Aparelho digestivo: clínica e cirurgia. $2^{\text {a }}$ ed. Rio de Janeiro: Medsi; 1996. p.617-22.

9. Tinoco RC. Cirurgia vídeo-laparoscópica na apendicite aguda. Medicina. 2001;127(3-4):20.

10. Williams MD, Miller D, Graves ED, Walsh C, Luterman A. Laparoscopic appendectomy, is it worth it? South Med. J. 1994; 87(6):592-8.

Fonte de financiamento: não há Conflito de interesse: não há Recebido para publicação: 10/12/2007 Aceito para publicação: 03/03/2008 